\title{
Effects of Diet and Behavior Therapy on Social and Motor Behavior of Retarded Phenylketonuric Adults: An Experimental Analysis
}

\author{
DAVID MARHOLIN II, ${ }^{(53)}$ ROBERT E. POHL III, R. MALCOLM STEWART, PAUL E. \\ TOUCHETTE, NANCY M. TOWNSEND, AND EDWIN H. KOLODNY
}

Boston University, The Eunice Kennedy Shriver Center for Mental Retardation, The Walter E. Fernald State

School, Massachusetts General Hospital, and Harvard Medical School, Boston, Massachusetts, USA

\begin{abstract}
Summary
The effects of a low phenylalanine diet on six retarded phenylketonuric adults were assessed. An ABA individual-subject design was used in experiment I to assess the effects of a low phenylalanine diet on social and motor behavior. Following a baseline during which the subjects ingested a normal phenylalanine diet (phase A), a low phenylalanine diet (phase B) was administered in a double blind fashion. Finally, the baseline condition (phase A) was reinstated (normal diet). The low phenylalanine diet resulted in few significant behavioral changes for those subjects with which proper methodologic controls were employed. However, for two of six subjects motor behavior, including stereotypy and tremor, seem to have ameliorated. In experiment II, applied behavior analysis techniques, including differential reinforcement of other behavior and time out, were combined to radically reduce the frequency of stereotypy and self-abuse exhibited by one of the six subjects of experiment $I$.
\end{abstract}

\section{Speculation}

Single-subject methodology borrowed from the experimental analysis of behavior revealed that a low phenylalanine diet was unsuccessful in producing significant desirable behavior changes in four adult retarded phenylketonuric subjects with proper experimental controls. However, when behavior therapy techniques were used with one of these same subjects, a rapid and dramatic reduction in stereotypy and self-abuse was observed.

Classic phenylketonuria (PKU) is a recessively inherited disease which causes severe mental retardation in untreated patients. In Massachusetts, it occurs with an incidence of 1 in 15,000 births (30). The liver enzyme phenylalanine-hydroxylase which converts phenylalanine to tyrosine is absent in these individuals. Phenylalanine and related metabolites (e.g., phenylpyruvic acid, phenyllactic acid, and $o$-hydroxyphenylacetic acid) accumulate in the blood, spinal fluid, and urine of untreated patients (29). In these patients, structural changes occur in the central nervous system, particularly in myelination, severely affecting mental performance. IQ scores below 50 are common (38).

PKU, when detected in infancy, is treated with a low phenylalanine diet with the amount of phenylalanine in the serum generally restricted to an amount below $10 \mathrm{mg} / \mathrm{dl}(17)$. When initiated with children under 6 months of age, damage to the central nervous system may be totally prevented $(8,18,26)$; even beginning a low phenylalanine diet as late as 2 years of age may be beneficial $(12,15,33)$. However, when begun after 2 years of age, the low phenylalanine diet cannot prevent severe retardation $(12,50)$. Although diet treatment started after 2 years of age is ineffective in preventing or correcting structural changes in the central nervous system, when administered to older PKU children and adults desirable changes in behavior have been reported $(2,3,12,16,18,19,42,47)$.

In perhaps the best controlled study, Frankenburg et al. (16) studied the social behaviors of 4- and 5-year-old PKU children not previously on any special diet. Social behaviors such as "lethargy," "timidity," "hyperactivity," and "irritability" were rated in the children's homes on a 95-item behavior rating scale by social workers and parents of the subjects. Following a baseline of ratings during a low phenylalanine diet, the diet was returned to normal according to a double blind protocol. Termination of the low phenylalanine diet and administration of the regular diet resulted in a reported increase in "naughtiness, lethargy, irritability, confusion, and restlessness." On the contrary, when the serum phenylalanine levels were decreased during the low phenylalanine diet phase, the children were more "friendly and appropriate."

Other studies also report improved behavior during administration of a phenylalanine-restricted diet. For example, in a study of older PKU adults (mean age $=22$ ), Bruhl et al. (12) reported that their subjects "improved in alertness and attention span" when placed on a low phenylalanine diet. Anderson et al. (2) further report that one of their subjects who was "excitable, hyperactive, noisy, and unmanageable" on a regular diet became "friendly and responsive" on the phenylalanine-restricted diet. However, contrary findings have also been reported. Hudson (22) and O'Flynn (39) noted no behavioral changes upon termination of a phenylalanine-restricted diet, and Solomons $e t$ al. (41) reported an "improvement in behavior" after termination of diet treatment.

One serious problem with all of the studies reporting behavioral changes as a function of diet treatment is the nonoperational nature of the response categories assessed. When global rating scales using nonspecific responses such as "irritability," "attentiveness," and "friendliness" are used to assess behavioral changes, the results can be easily confounded by the observers' expectations regarding the effects due to treatment $(37,40,41$, 44). Such a criticism is especially valid when studies are not conducted in a double blind manner, which was true of almost all those reporting behavioral changes as a function of diet treatment. For example, biasing information was found to be directly responsible for positive behavioral changes following diet therapy in a study conducted by Habraeus et al. (19). In a pilot study, it was reported that adult PKU subjects exhibited less "agitated behavior" when placed on a low phenylalanine diet. However, the observers knew the conditions and objectives of the study. When the study was replicated using a double blind procedure, the same behavioral changes were not observed. In a study conducted by Siegel et al. (44), it was found that only rating scales constructed of discrete and readily identifiable measures which can be reliably counted were resistant to biasing effects. Thus, it is crucial that research efforts to assess behavior 
change as a function of diet therapy use rating scales comprised of discrete and measurable items with interobserver reliabilities provided $(9,27,34)$.

While the effect of diet therapy after age 2 on severely retarded PKU individuals is unclear, behavior modification techniques have been successful in affecting a variety of social and motor behaviors of the severely and profoundly retarded $(7,10)$. One procedure that has proven particularly effective in the reduction of a variety of undesirable behaviors is termed differential reinforcement of other behavior (DRO). A DRO schedule of reinforcement allows for the reinforcement of any behavior an individual emits except a particular one (14). Thus, the DRO schedule specifies the behavior which is to go unreinforced rather than the one which is increased in frequency. The result of such a schedule of reinforcement is a decrease in the frequency of a particular specified behavior. For example, a DRO procedure has successfully reduced seizures (51), verbal outbursts (11), and aggressive (11) and self-injurious behavior (13) of retarded individuals.

The purpose of the present study was to assess the effect of a low phenylalanine diet on the social and motor behavior of severely retarded PKU adults. Precise behavioral observation techniques were adopted. An attempt was made to present diet therapy in a double blind fashion. Finally, the effects of diet therapy were contrasted with behavior modification procedures.

\section{MATERIALS AND METHODS}

A within-subject reversal or ABA design common to applied behavior analysis was used to assess the effects of diet therapy. The ABA or reversal design demonstrates the effect of treatment on an individual subject by alternately presenting and removing it so that its influence may be observed over time. The design begins with assessment of baseline rates of behavior ( $A$ phase). Baseline data assess the initial pretreatment level and predict what levels of behavior are likely to be in the immediate future if no treatment were provided.

When the baseline shows stability over time, treatment is implemented (B phase). Treatment is continued until behavior changes and reaches a stable level. At this point, treatment usually is withdrawn and baseline conditions (A phase) are reinstated. Typically, behavior returns or approaches baseline levels of performance. The purpose of alternating phases is to establish the cause of behavior change. This is accomplished by assessing behaviors under baseline and treatment conditions. Data in each phase describe behavior under a particular set of conditions (e.g., low phenylalanine diet or regular diet) and predict what behavior would be like if these conditions remained in effect. When behavior deviates at or shortly after a change in treatment conditions, this suggests that the change from baseline to treatment or from treatment to baseline was casual. If, after the original conditions are reinstated, the original level of behavior is recovered, it is likely that the intervention led to change.

Within-subject designs are discussed in more detail elsewhere $(6,21,28,43)$. These designs have been recently used in clinical drug studies with humans (e.g., 4, 31, 36). Within-subject research methodologies are particularly useful in studying effects of diet change on behavior, because an inherent problem in assessing effects of metabolic intervention lies in the fact that each person is likely to react to the presence or absence of specific amounts of phenylalanine on a highly individual basis.

\section{EXPERIMENT I}

\section{SOCIAL AND PHYSICAL SETTING}

This study was conducted at the Walter E. Fernald State School in Waltham, MA. Each subject participating in the study resided on a ward containing an average of 18 residents. Ward programs included recreational activities, individual tutorials, and periodic skill training sessions based upon behavior modification procedures. No major treatment procedures were initiated or terminated during the course of the study.

\section{SUBJECTS}

Six adult male and female residents were selected from a group of 15 residents with PKU previously studied (48).

Subject 1 was a 25 -year-old female. Her social quotient on the Vineland Social Maturity Scale was 14 . Between ages 5 and 7 years she received a phenylalanine-restricted diet. Subject 2 was a 53-year-old male. His IQ was 30 as measured by the Stanford-Binet Intelligence Scale. He was diagnosed as having PKU at the age of 13 . Subject 3 was a 42 -year-old male. He was diagnosed as having PKU at the age of 15 . The Vineland Social Maturity Scale yielded a social quotient of 4 . Subject 4 was a 27-year-old female. Her IQ measured by the Stanford-Binet was 6 . Because of poor muscle control and flacid lower extremities, she was confined to a wheelchair. At age 3 she was found to have PKU and was placed on a low phenylalanine diet until she was 5 . Subject 5 was a 19-year-old male with a social quotient of 36 as measured by the Vineland Social Maturity Scale. He was diagnosed as having PKU at age 4.5 years, and he was placed at that time on a low phenylalanine diet which was continued until age 9 . Subject 6 was a 50-year-old male. His social quotient was 13 as measured by the Vineland Social Maturity Scale. Informed consent was obtained from the guardians and families of each of the subjects participating in the study in accordance with Department of Health, Education and Welfare and the Commonwealth of Massachusetts ethical and legal standards.

\section{RESPONSE DEFINITION AND DATA COLLECTION PROCEDURES}

Data were collected on a subset of 19 behaviors in a free-field unstructured ward setting for subjects $1,3,4,5$, and 6 . Subject 2 was observed in a structured kitchen setting. A coding system modeled after those described by Bijou et al. (9) and Lichstein and Wahler (32) was employed in this research. Table 1 outlines the behavior categories used in the study. A total of 19 categories were employed. Of these, 18 described behaviors of the target subjects (response events). A final category, slash (SL) indicated that no scorable response occurred during the defined interval. A subset of the 19 categories was chosen for each subject based upon prestudy observations of their operant rates of performance. Selected behaviors were recorded based upon their occurrence during daily 15 -min observation periods. Each 15-min observation period was divided into 6010 -sec observation intervals (i.e., observe for $10 \mathrm{sec}$, record for $5 \mathrm{sec}$, repeat). Multiple behaviors could be recorded in any one interval. Behaviors occurring during the 5 -sec record intervals were not scored.

Trained observers remained as inconspicuous as possible (approximately $15 \mathrm{~m}$ from the subjects). They avoided making eye contact with the target subject and ignored any instances of the subject approaching or otherwise interacting with them. Two observers simultaneously gathered reliability data on the same subject on the average of once in every five sessions.

The interobserver reliability of these data was computed separately for each 10 -sec period using I-I and S-I reliability calculations (20). In I-I or interval-by-interval reliability an agreement is any interval in which both observers recorded that the response occurred during the interval, or in which both observers recorded that the response did not occur during the interval. Disagreements are intervals in which only one observer reported that the response occurred. The percentage of agreement equals the number of agreements divided by the sum of such agreements that a particular behavior occurred plus disagreements per 15 -min sessions and multiplied by 100 to obtain a percentage agreement.

The second reliability calculation is referred to as the S-I, or scored interval method. In S-I agreement, all intervals in which 
Table 1.Description of category codes

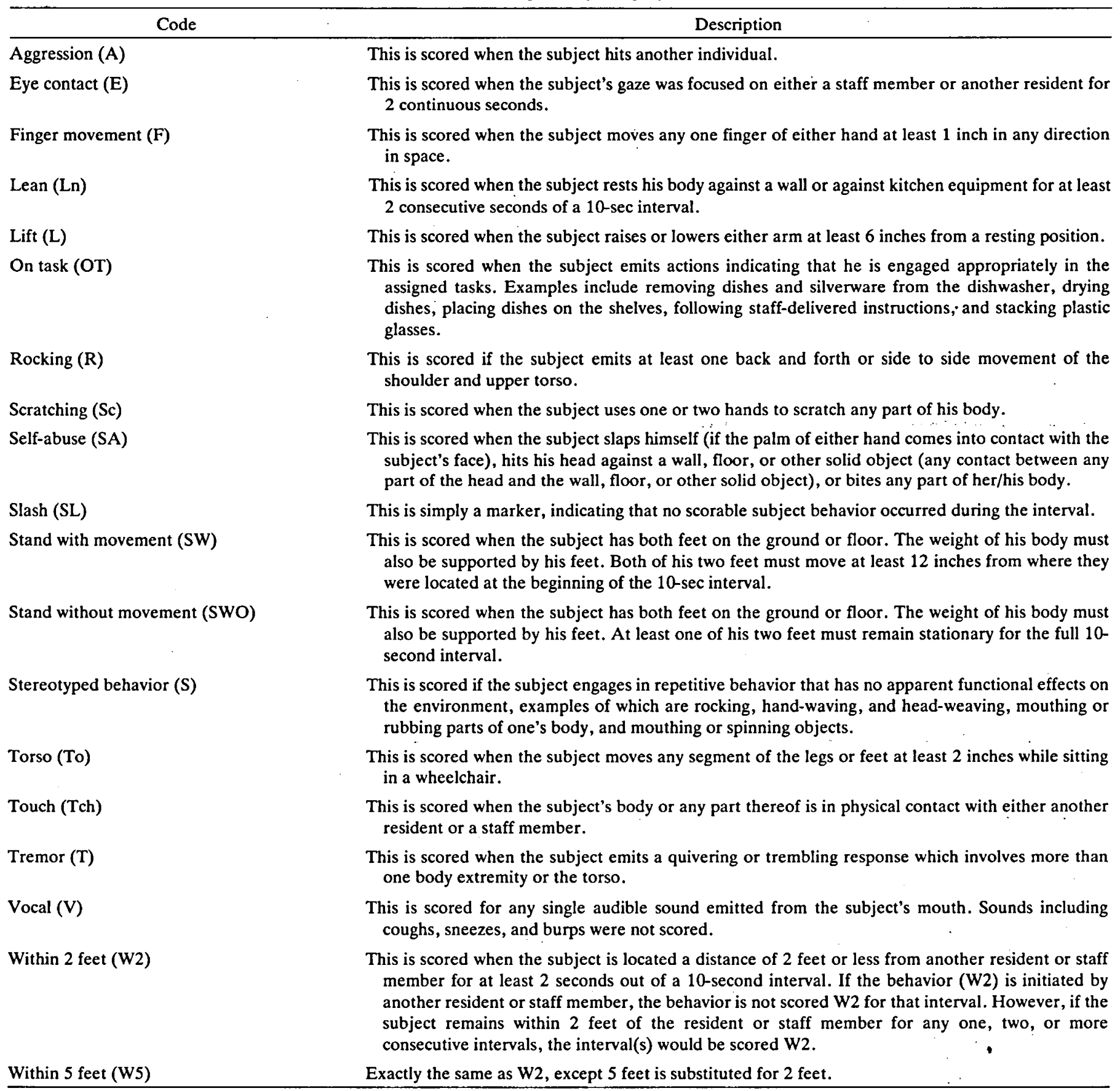

neither observer scores the behavior occurring are ignored in calculating agreement scores. Only an interval in which both observers recorded the occurrence of a behavior are counted as agreements. The S-I method is a more stringent measure of reliability. However, at relatively low frequencies of behavior, S-I scores become highly variable. For example, if one observer recorded a response as occurring in only one interval and the other recorded it as occurring in no intervals, their agreement is $0 \%$ as calculated by the S-I method, whereas a change of just one interval on the second observer's data sheet would make the agreement $100 \%$. At the same time, one disagreement as noted above would result in a $98 \%$ agreement (based on 60 intervals) as calculated by the I-I method.

The range of I-I and S-I reliability percentages summed across the six subjects completing the study is presented in Table 2. Overall, mean reliabilities of $93.8 \%$ and $74.1 \%$ were attained by I-I and S-I methods, respectively.

\section{PROCEDURE}

Each subject's daily levels of social and motor behavior were observed both on and off a low phenylalanine diet. A partial double-blind ABA design was used where $A$ represented regular institutional diet and $\mathrm{B}$ represented a low phenylalanine diet. The design of the study included the following three phases.

Phase 1: Baseline. All six subjects were observed for a mean of 14 days (range $=7-22$ days) to evaluate social and motor behavior when they were on a regular institutional diet. Each subject was observed for $15 \mathrm{~min}$ each day, using a time-sampling procedure as described by Marholin and Bijou (34). The 15-min observation allowed for the evaluation of a predetermined subset of the 19 categories of behavior listed in Table 1. All subjects were observed at approximately the same time each day, 4 or 5 days a week (excluding Saturdays and Sundays), from approximately 7:00 AM to 9:00 AM. 
Table 2. Range and means of reliability percentages (scoredinterval and interval-by-interval calculations)

\begin{tabular}{|c|c|c|c|c|}
\hline \multirow[b]{3}{*}{ Code } & \multicolumn{4}{|c|}{ Interobserver reliabilities } \\
\hline & \multicolumn{2}{|c|}{ S-I method } & \multicolumn{2}{|c|}{ I-I method } \\
\hline & Range & Mean & Range & Mean \\
\hline Aggression (A) & $100-100$ & 100 & $100-100$ & 100 \\
\hline Eye contact (E) & $25-76$ & 60 & $85-90$ & 88 \\
\hline Finger movement $(F)$ & $33-100$ & 56 & $90-97$ & 93 \\
\hline Lean $(\mathrm{Ln})$ & $62-86$ & 74 & $91-97$ & 94 \\
\hline Lift (L) & $63-100$ & 81 & $92-100$ & 96 \\
\hline On task (OT) & $87-100$ & 95 & $94-100$ & 98 \\
\hline Rocking (R) & $42-100$ & 71 & $90-100$ & 91 \\
\hline Scratching (Sc) & $63-100$ & 84 & $80-97$ & 87 \\
\hline Self-abuse (SA) & $88-100$ & 97 & $83-100$ & 94 \\
\hline Stand with movement (SW) & $32-80$ & 74 & 93-98 & 96 \\
\hline Stand without movement (SWO) & $50-97$ & 71 & $97-98$ & 98 \\
\hline Stereotype (S) & $50-75$ & 66 & $84-98$ & 93 \\
\hline Torso (To) & $33-100$ & 44 & $80-99$ & 88 \\
\hline Touch (Tch) & $59-100$ & 94 & $90-100$ & 95 \\
\hline Tremor (T) & $24-85$ & 45 & $88-100$ & 96 \\
\hline Vocal (V) & $25-92$ & 48 & $90-100$ & 97 \\
\hline Within 2 feet (W2) & $74-89$ & 83 & $76-98$ & 88 \\
\hline Within 5 feet (W5) & $60-98$ & 91 & $87-98$ & 93 \\
\hline
\end{tabular}

Table 3. Individual subject data: weight, caloric restrictions, and daily phenylalanine intake

\begin{tabular}{cccccc}
\hline & & & & & \\
Subject & $\begin{array}{c}\text { Initial } \\
\text { wt, } \mathrm{kg}\end{array}$ & $\begin{array}{c}\text { Final } \\
\text { wt, } \mathrm{kg}\end{array}$ & $\mathrm{Wt}, \mathrm{kg}$ & $\mathrm{Cal}$ & $\begin{array}{c}\text { Dietary } \\
\text { Phenyla- } \\
\text { lanine, } \\
\mathrm{mg} / \mathrm{kg}\end{array}$ \\
\hline 1 & 45.0 & 47.0 & +2.0 & 3650 & 11.1 \\
2 & 63.0 & 65.5 & +2.5 & 2850 & 7.9 \\
3 & 48.0 & 45.0 & -3.0 & 3000 & 11.4 \\
4 & 42.5 & 43.0 & +0.5 & 2650 & 8.8 \\
5 & 64.0 & 63.0 & -1.0 & 3825 & 8.7 \\
6 & 65.5 & 63.0 & -2.5 & 1 & 1 \\
\hline
\end{tabular}

${ }^{1}$ Because Subject 6 lost weight during the study and failed to eat, stable caloric and phenylalanine measures were unavailable.

Phase 2: Low Phenylalanine Diet. The regular institutional diet was discontinued, and a low phenylalanine diet modeled after that used by the Boston Children's Hospital Medical Center PKU Clinic consisting of Lofenalac (Mead Johnson \& Co.) supplemented with appropriate fruits, vegetables, and low phenylalanine bread and milk was instituted (see Table 3). Five of the six subjects received the low phenylalanine diet for a mean of 56 days, with a range of from 53-59 days. Subject 6 received the low phenylalanine diet for 18 days. Fifteen-minute time-sample observations of the subjects' behaviors were continued.

Phase 3: Baseline. Each subject except subjects 5 and 6 was taken from his or her place of residence and placed in the hospital of the Eunice Kennedy Shriver Center for two 1-day periods. The two 1-day periods were separated by 7 days during which no time-sampled observations were taken. During each subject's stay in the hospital, biochemical studies were carried out (49) which included a phenylalanine loading each time a subject was taken from his building. The phenylalanine loading subsequently brought each subject's serum phenylalanine level up to that observed prior to the low phenylalanine diet. After each subject's second hospital visit, regular institutional meals were reinstituted, and 15-min time-sampled observations of their behavior were continued. Each subject was observed for a mean of 15 days, with a range from 10-21 days.

Double Blind. Although building staff knew that the subjects were to be placed on and off the low phenylalanine diet, the study was designed so that the observers would be blind as to the onset and termination dates of the low phenylalanine diet. The double-blind was partially broken when signs were posted on the wards of subjects 2 and 3 indicating the onset of the "special diet" and warning staff not to allow the subjects to have regular institutional food. However, observers of behavior were not aware of the precise termination date of the low phenylalanine diet which preserved a portion of the double-blind relative to subjects 2 and 3 . The observers collecting data were blind as to the onset and discontinuation of the low phenylalanine diets of subjects $1,4,5$, and 6 .

Blood Studies. Peripheral venous blood was drawn twice weekly for plasma amino acid determinations. Serial determinations of phenylalanine were made by a manual flurometric assay (1). These determinations were made by an individual unaware of the results of the behavioral assessment procedures.
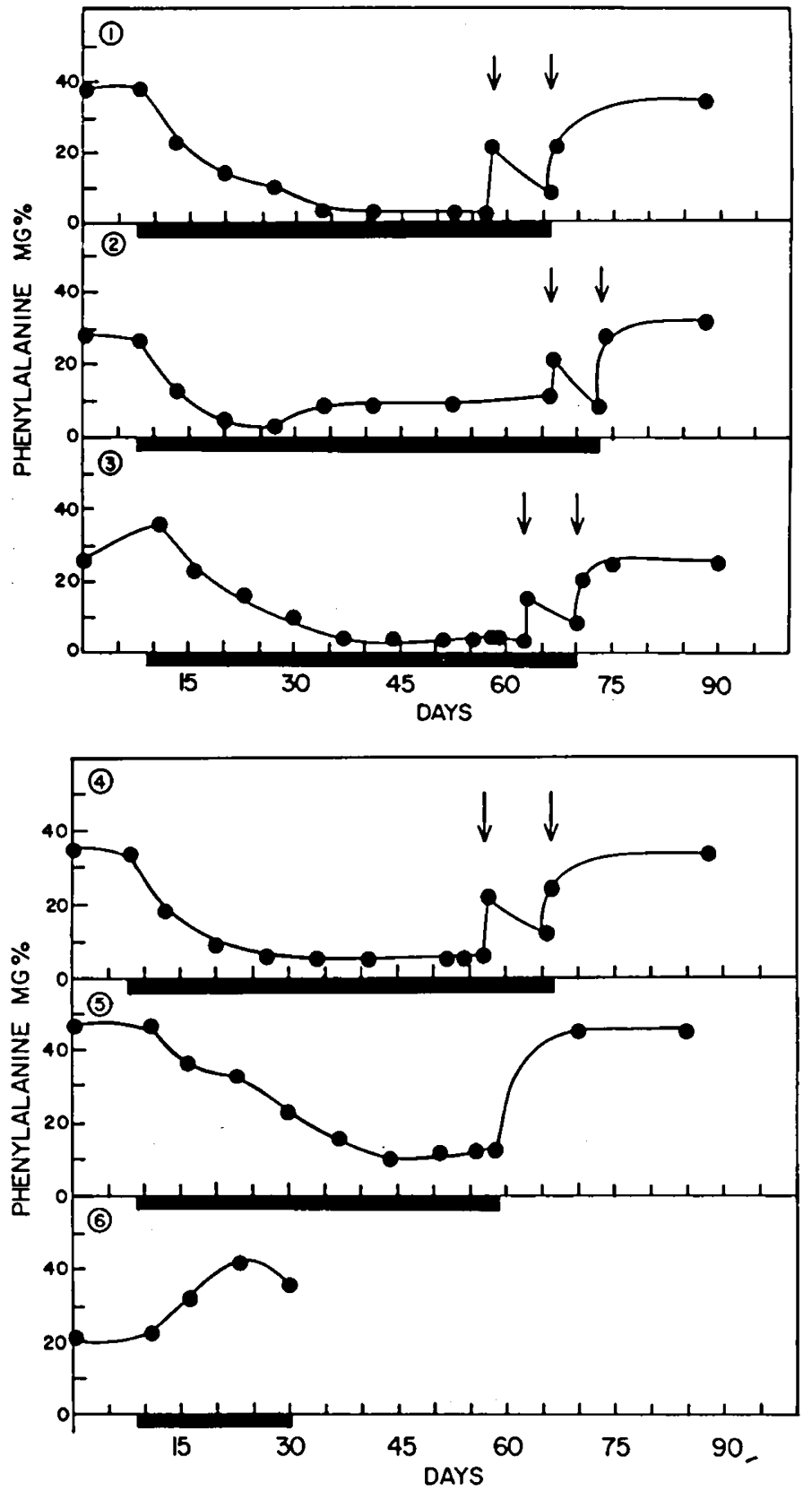

Fig. 1. Time course for serum phenylalanine (Phe) levels in phenylketonuric adults. Low phenylalanine diet treatment occurred during periods indicated by solid bars below day lines. Note that five subjects had a reduction of Phe to less than $10 \mathrm{mg} / 100 \mathrm{ml}$ while one subject (patient 6) had an increase to $40 \mathrm{mg} / 100 \mathrm{ml}$. Arrows represent phenylalanine loadings for biochemical studies. 
S-1

BEHAVIORAL RESPONSE TO LOW PHENYLALANINE DIET
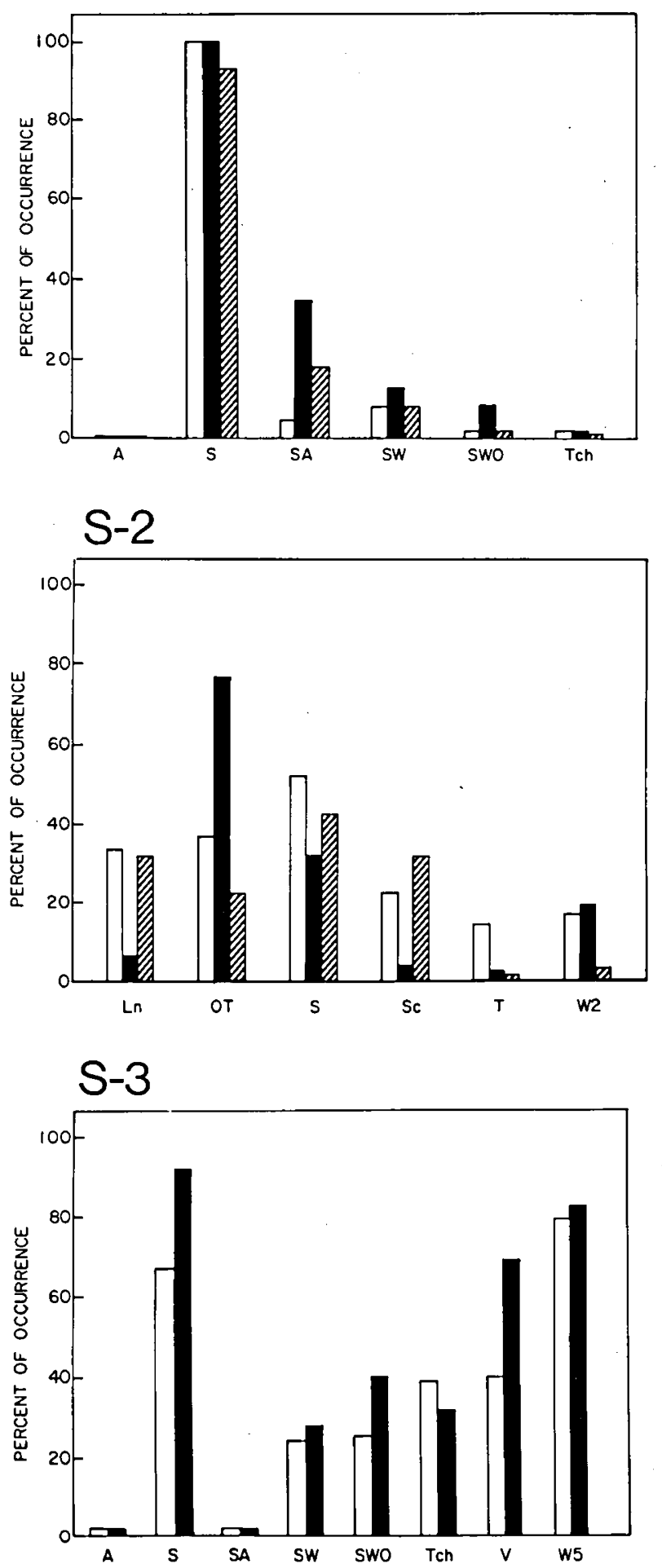

S-4

BEHAVIORAL RESPONSE TO LOW PHENYLALANINE DIET
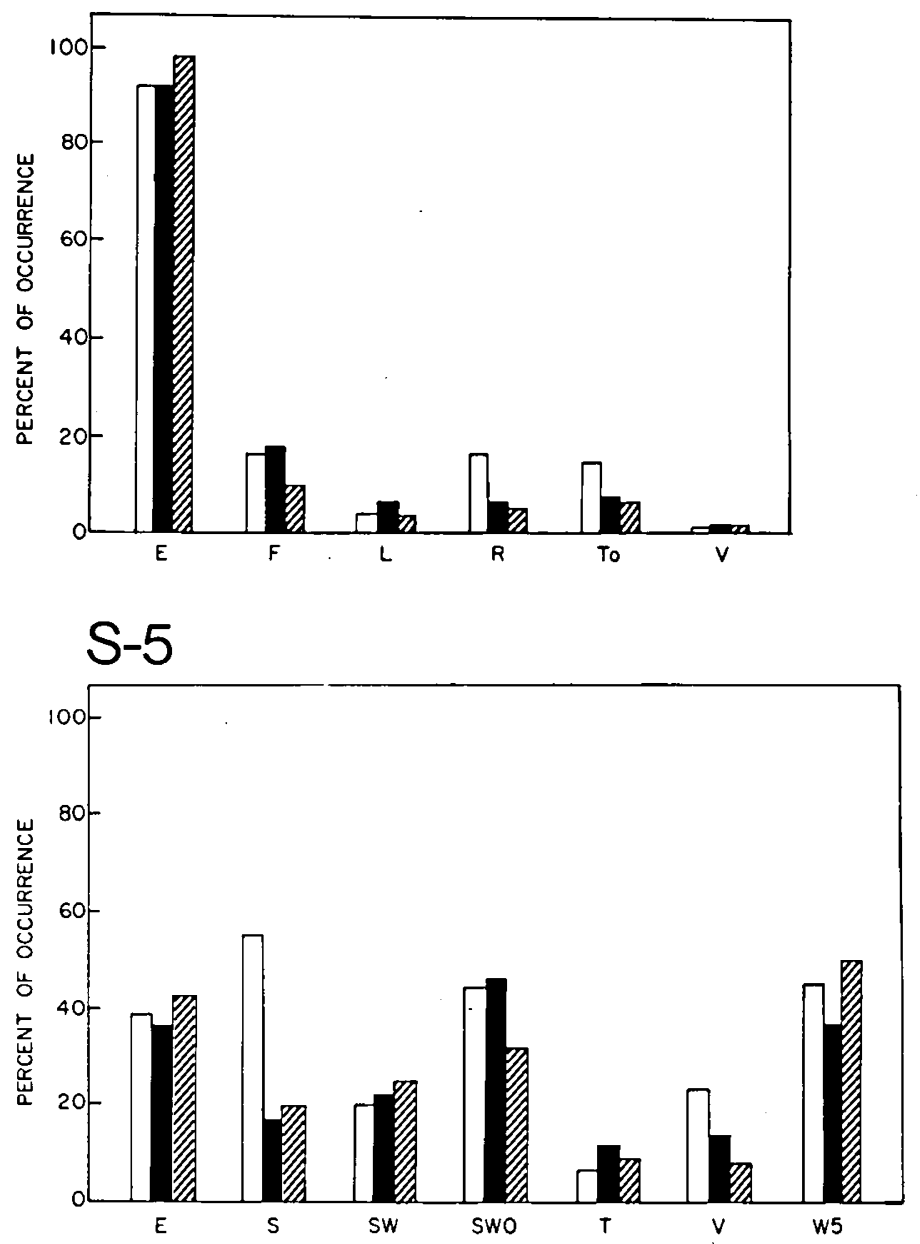

S-6

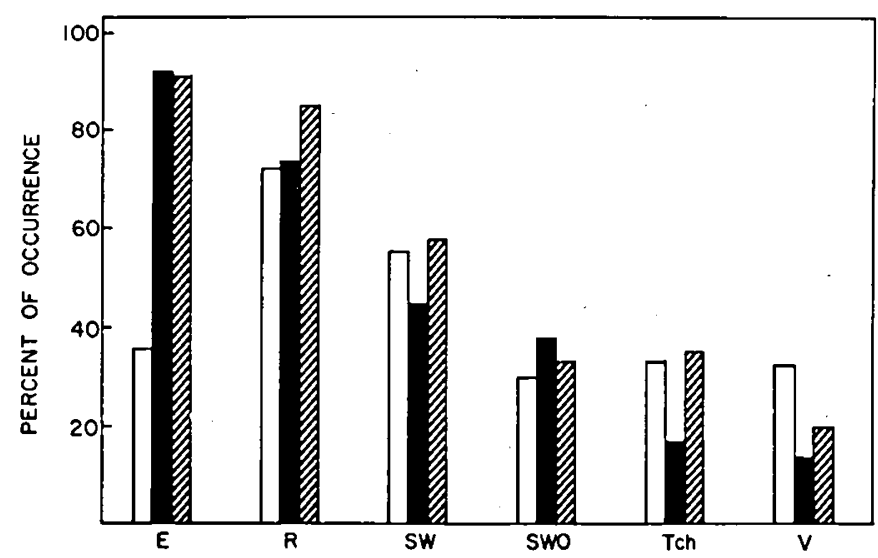

Fig. 2. Bar graphs of behavioral responses to low phenylalanine diet plotted as percent of occurrences during all experimental conditions: $\square$, baseline; $\square$, low phenylalanine diet; and $\mathbb{Z}$, return-to-baseline. Symbols are as follows: A, aggression; E, eye contact; F, finger movement; Ln, lean; L, lift; OT, on task; R, rocking, Sc, scratching; SA, self-abuse; SL, slash; SW, stand with movement; SWO, stand without movement; S, stereotyped behavior; To, torso; Tch, touch; T, tremor; V, vocal; W2, within 2 feet; and W5, within 5 feet.

\section{RESULTS}

Serum Phenylalanine Levels. The serial determinations of phenylalanine are summarized in Figure 1. Serial monitoring revealed that the low phenylalanine diet was effective in lowering the serum phenylalanine levels in subjects 1 through 5 from values ranging from 35 to $48 \mathrm{mg} / \mathrm{dl}$ during Phase 1 (initial baseline) to values ranging from $3-10 \mathrm{mg} / \mathrm{dl}$ on the low phenylalanine diet (Phase 2). Finally, reinstatement of the normal institutional diet (Phase 3 ) resulted in phenylalanine values ranging from $33-45 \mathrm{mg} / \mathrm{dl}$. Subject 6 's phenylalanine level increased from $21-40 \mathrm{mg} / \mathrm{dl}$ while on the low phenylalanine diet.

Social and Motor Behavior. The overall effect of a low 
phenylalanine diet on the social and motor behavior of the six subjects is summarized in Figure 2.

Subject 1. Six behaviors were assessed for subject 1 . As can be seen in Figure 2, the only systematic changes were observed for self-abuse and stand without movement. Self-abuse occurred more frequently during the diet treatment phase than during either of the baseline conditions. The diet failed to significantly affect any of the other four behaviors measured.

Subject 2. Of the six behaviors assessed for subject 2 , four were systematically affected by the low phenylalanine diet. This subject was more frequently on task and less frequently engaged in leaning, scratching, and stereotyped behavior during the period he received the low phenylalanine diet in comparison to both baseline phases. Tremor dropped from a mean of $14 \%$ during phase 1 to a mean of $2 \%$ during the diet treatment condition (phase 3 ) and did not reoccur up to 6 weeks after the low phenylalanine diet was discontinued.

Subject 3. Eight behaviors were assessed of subject 3. As can be seen in Figure 2, institution of the low phenylalanine diet resulted in an increased frequency of vocal and stereotyped behavior and stand with movement. There was no return-tobaseline (phase 3 ) condition.

Subject 4. Of the six behaviors assessed of subject 4, no systematic changes were observed throughout the study.

Subject 5 . Of the seven behaviors assessed for subject 5 , only stereotypy changed significantly throughout the duration of the study. It decreased from a mean pretreatment occurrence of $62 \%$ to a mean of $17 \%$ during the low phenylalanine diet condition, where it remained after the low phenylalanine diet was discontinued (mean $=20 \%$ ).

Subject 6 . Of the six behaviors assessed for subject 6 , the only behavior in which a systematic change occurred was touch. Subject 6 engaged in slightly less touching behavior during the diet treatment condition when his serum phenylalanine level was elevated as compared to off-diet treatment conditions. Although subject 6 engaged in more eye contact (E) during the diet treatment condition ( $92 \%$ ) as compared to the pretreatment baseline $(36 \%)$, eye contact failed to decrease when the low phenylalanine diet was terminated. None of the other four behaviors assessed changed systematically during the entire duration of the study.

\section{DISCUSSION}

The results indicate that the social and motor behavior of previously untreated PKU retarded adults is not consistently changed by a low phenylalanine diet. Of the six subjects who were chosen for the study, the low phenylalanine diet lowered the serum phenylalanine levels for five of the subjects. For three of these five subjects (subjects 1,4, and 5) only minor behavioral changes were observed. Although significant behavioral changes were noted for subjects 2 and 3 , methodologic problems limit their interpretation. Finally, with the one subject in which the low phenylalanine diet resulted in an increased rather than a decreased serum phenylalanine level (subject 6 ), no significant, systematic behavioral changes were noted.

Although slight increases in stand with (SW) and without (SWO) movement were observed with subject 1 , the only significant behavioral change as a function of the low phenylalanine diet was observed with self-abuse. Because there was a higher frequency of self-abusive behavior during the diet treatment condition as compared to both baseline conditions, the behavioral change might be seen as a negative side effect of the "special diet." Moreover, of the 13 social and motor behaviors assessed of subjects 4 and 5 , only the stereotypy of subject 5 changed significantly during the study. Stereotypy decreased dramatically when the low phenylalanine diet was instituted, and remained so for several weeks after the diet was terminated. The same effect was noted with subject 2 for tremor, a related motor behavior. It is possible that in the case of changes in motor behavior physiologic effects of the low phenylalanine diet, perhaps including effects on central neurotransmitters last beyond the termination point of the low phenylalanine diet itself. Therefore, it might be suggested that a longer return-tobaseline condition may be necessary to see the reversal.

Although significant social behavioral changes were observed with subjects 2 and 3 , several methodologic problems must be noted. First, because the double blind was partially broken after signs were posted when the low phenylalanine diet was initiated, the observed changes in behavior must be interpreted with caution (19) even though operationally defined and reliably observed behavioral categories were used (44). Second, because of an unplanned change in subject 3's environment, no returnto-baseline condition was initiated. Therefore, there is no clear causal relationship between the increases in vocal (V), stand with movement (SW), and stereotypy (S) and the diet treatment condition $(5,43)$. Third, since subject 2 worked and was observed in the kitchen during meal times, building staff were particularly careful to provide him with tasks during the diet treatment condition to minimize the likelihood of his picking up and ingesting normal foods rich in phenylalanine available in the kitchen. Although no data were recorded, building staff seemed to interact more frequently with subject 2 during the diet treatment condition. Thus, it is not surprising that subject 2 's frequency of on-task behavior (OT) increased when the low phenylalanine diet was initiated and decreased when the diet was terminated because of increased opportunities for engaging in specific kitchen tasks. Moreover, the systematic decrease in leaning (Ln), scratching (Sc), and stereotypy (S) during the diet treatment condition would be expected, because each of these behaviors are incompatible with being on task (OT). Although the observed behavior changes during the diet treatment condition may have been confounded by systematic differences in staff interaction and/or task opportunity, it is clear that subject 2's behavior dramatically changed. However, the change cannot necessarily be attributed to the effect of the low phenylalanine diet itself.

In summary, it appears from the data that few significant desirable behavioral changes occurred as a function of the low phenylalanine diet for those subjects with which proper methodological controls were employed (subjects $1,4,5,6$ ). It is noteworthy that no significant, systematic changes were observed as well for subject 6 , whose serum phenylalanine level was increased during the diet treatment condition, as well as for subjects 1,4 , and 5 , whose serum phenylalanine levels were successfully decreased during the diet condition. Therefore, it appears that neither a controlled increase nor decrease in serum phenylalanine levels is correlated with systematic social behavior change, although motor behavior including tremor and stereotypy seem to have ameliorated in two of six subjects. It is also clear that with at least two subjects, changes in social behavior can be achieved, but, because of the methodologic problems, the changes cannot be attributed solely to the lowering of their serum phenylalanine levels.

\section{EXPERIMENT II}

Because behavior modification techniques have been successful in affecting numerous social and motor behaviors of the severely and profoundly retarded $(7,10)$, they were used as a contrasting treatment to diet therapy with subject 1 . Subject 1 exhibited an extremely high frequency of stereotyped behavior as well as some self-abusive behavior during all phases of experiment 1 . No noticeable change in stereotyped behavior occurred during the diet manipulation but a reliably higher frequency of self-abusive behavior was observed. She was chosen for the behavior modification study on the basis of an extremely high frequency of stereotyped behavior observed during experiment $\mathrm{I}$.

\section{SETTING}

Treatment sessions took place in a large, locked ward approximately $18 \mathrm{~m} \times 6.5 \mathrm{~m}$. The ratio of mental health assistants to residents was generally $1: 8$. 


\section{RESPONSE DEFINITIONS AND DATA COLLECTION PROCEDURES}

Body rocking and repetitious hand and finger movements were the stereotyped behaviors recorded for subject 1 ; striking herself in the face or banging her head on the wall were the selfabusive behaviors recorded. In addition, five other social and motor behaviors of this subject reported in experiment I continued to be recorded. Daily. 15-min time-sampled observations were taken by a trained observer. Interobserver reliability computed by the I-I method averaged $92 \%$ with a range from $90-93 \%$.

\section{PROCEDURE}

An ABCADAD within-subject design was used to assess the effects of treatment conditions on the subject's behavior.

Phase I: Baseline $(A)$. Data were recorded for 10 days on stereotyped behavior and six other social and motor behaviors. No systematic reinforcement was delivered during this baseline phase.

Phase 2: Treatment 1 (B). On day 11 , treatment 1 was instituted. Treatment included a combination of two behavioral techniques: (1) differential reinforcement of other behavior (DRO) and, (2) time out from reinforcement. The subject was reinforced whenever she engaged in any behavior other than stereotypy or self-abuse for 10 successive seconds. If the subject engaged in any instances of stereotypy of self-abuse during any part of the 10-sec interval, she was not reinforced. The subject was reinforced by the trainer with grape juice delivered from a spray bottle preceded by verbal praise (e.g., "Good standing." "Quiet hands."). Subject 1 continued to receive reinforcement at the end of each succeeding 10-sec interval as long as selfabusive or stereotyped behavior did not occur.

If any instances of stereotyped or self-abusive behavior occurred, time out was immediately instituted. That is, the trainer immediately turned and walked approximately $3 \mathrm{~m}$ away from the subject and remained with her back to her for $10 \mathrm{sec}$. At the end of the 10-sec time out, the trainer returned to the subject's immediate presence. If she was observed emitting stereotyped or self-abusive behaviors, time out was reinstated. However, if no stereotyped or self-abusive behaviors were emitted, the trainer established eye contact with the subject and reinforced her at the end of $10 \mathrm{sec}$ as long as no instance of self-abusive or sterotyped behaviors were observed. Treatment 1 lasted 11 days.

Phase 3: Treatment $2(C)$. Treatment 1 was terminated on day 22 , and treatment 2 was begun. Treatment 2 consisted of six sessions using a procedure identical to that of treatment 1 with one exception. Five successive seconds of no occurrences of selfabusive or stereotyped behaviors were now required for reinforcement rather than the $10 \mathrm{sec}$ required for reinforcement in treatment 1 .

Phase 4: Baseline (A). On day 28, treatment 2 was discontinued, and baseline conditions were reinstated for five sessions. DRO and time out procedures were discontinued. No reinforcement was delivered.

Phase 5: Treatment $3(D)$. Treatment 3 began on day 33. In phase 5 ice cream was used as the primary reinforcer. Five treatment sessions were held with a 10 -sec DRO and time out.

Phase 6: Baseline (A). On day 38, DRO and time out procedures were discontinued and baseline conditions were reinstated. The trainer did not deliver systematic social or primary reinforcement during these baseline sessions. Phase 6 lasted for 2 days.

Phase 7: Treatment 3 (D). On day 40, treatment 3 was reinstated. Phase 7 lasted for 6 days.

\section{RESULTS}

Figure 3 shows the mean percentage of stereotyped behavior exhibited by subject 1 during all phases of experiment II.

Phase 1: Baseline (A). Baseline data reveal a mean percentage of $86 \%$ stereotyped behavior during the 10 baseline sessions. The range of occurrence over the 10 days was $55-100 \%$.

Phase 2: Treatment $1(B)$. Implementing treatment 1 resulted in a slight mean decrease in stereotyped behavior to a mean of $75 \%$, with a range from $40-95 \%$.

Phase 3: Treatment 2(C). During the six sessions of treatment 2 , stereotyped behaviors occurred during a mean of $79 \%$ of the 15 -min sessions with a range from $50 \%$ to $99 \%$.

Phase 4: Baseline $(A)$. During the five baseline sessions, a mean of $79 \%$ (range $=60-93 \%$ ) of the recording intervals included stereotyped behaviors.

Phase 5: Treatment 3 (D). Implementing treatment 3 resulted in an average of $37 \%$ stereotyped behavior (range $=16-59 \%$ ),

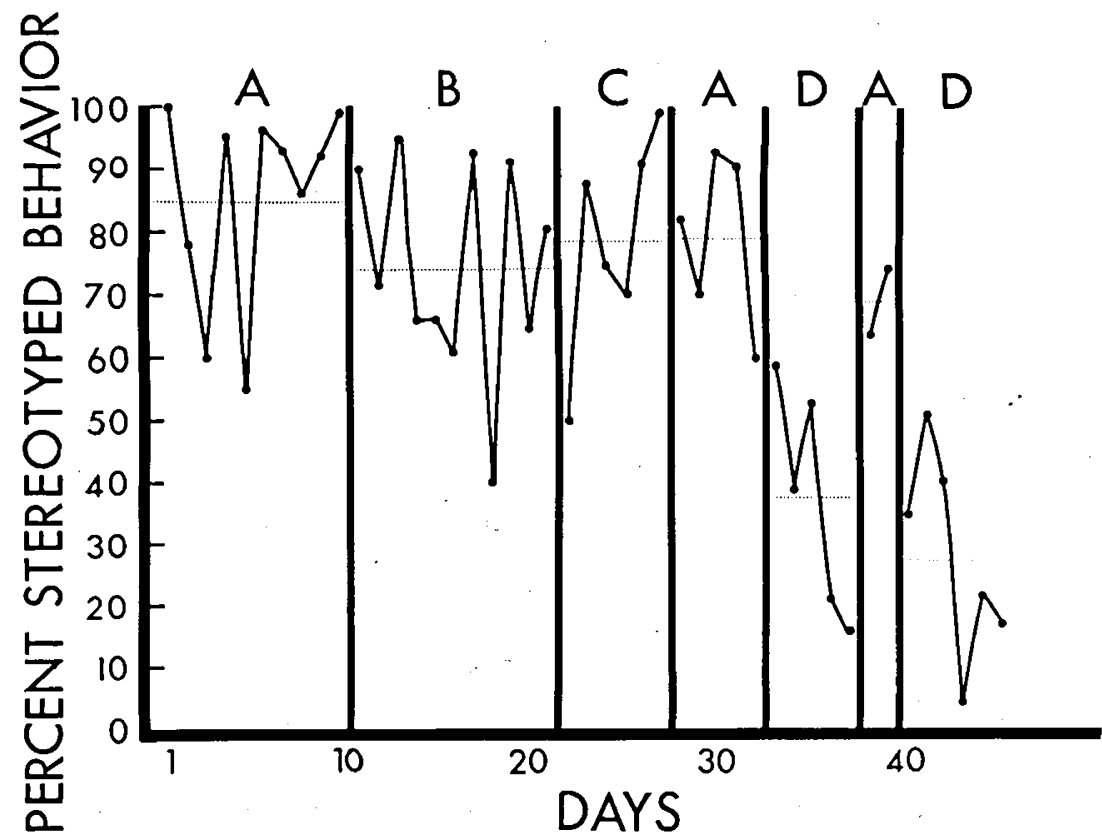

Fig. 3. Percentage of occurrence of stereotyped behavior. A: baseline; B: 10-sec DRO (grape juice and social reinforcement) and 10-sec time out; C: 5-sec DRO (grape juice and social reinforcement) and 10-sec time out; D: 10-sec DRO (ice cream and social reinforcement) and 10-sec time out. 
a dramatic mean decrease of $40 \%$ from the previous baseline condition.

Phase 6: Baseline $(A)$. Reinstatement of the baseline phase resulted in a dramatic increase over treatment 3 conditions to a mean of $69 \%$ stereotyped behavior (range $=64-74 \%$ ).

Phase 7: Treatment $3(D)$. Finally, data revealed that reinstating treatment 3 procedures resulted in a significant decrease from the mean of the preceding baseline phase to $28 \%$ (range $=4-51 \%$ ).

In addition to stereotypy, six other social and motor behaviors were recorded during experiment II. They included stand with (SW) and without (SWO) movement, vocalization (V), aggression (A), and touch (Tch). Self-abusive behaviors declined to zero during both treatment 3 conditions (i.e., phases 5 and 7) as compared to means of $22 \%, 18 \%$, and $20 \%$ during the three baseline conditions and $17 \%$ and $22 \%$ during treatments 1 and 2 . The other five behaviors recorded did not systematically change throughout the study.

\section{DISCUSSION}

Although a low phenylalanine diet administered during experiment I proved unsuccessful in affecting the behavior of subject 1 , a behavioral treatment technique was successful in dramatically reducing stereotypy and self-abusive behavior. The data show that with a functional reinforcer, subject 1's stereotyped behaviors were rapidly reduced in frequency (phase 5). Data from the reversal sessions (phase 6) demonstrate that in the absence of DRO and time out procedures, subject 1 's incidence of stereotyped behavior increased. When treatment was reinstated (phase 7), the percentage of stereotyped behavior again dropped. The failure to reduce the incidence of self-abuse and stereotypy during treatments 2 and 3 was probably due to the failure of grape juice to act as a functional reinforcer. When ice cream was substituted for grape juice as a primary reinforcer during treatment 3 conditions, a strong and rapid reduction in stereotypy was observed.

During phase 5 (first introduction of treatment 3), subject 1 emitted behavior which might be described as self-controlling responses (45). Subject 1 initially held the trainer's arms with a strong grip, squeezing them rhythmically. During later sessions of phase 5, her grip strength was noticeably reduced and the squeezing was absent. During the final session of phase 5 , she merely rested her hands lightly on the trainer's arms. She was also observed clenching her hands behind her back, a response incompatible with stereotypic behavior involving her hands. A similar sequence of self-controlling responses were observed when treatment 3 was continued in phase 7 . Although selfcontrol procedures have been extensively researched with adults $(24,25)$ and children of normal intelligence $(23,35)$, selfcontrol procedures need to be empirically investigated with the retarded.

In summary, a single-subject methodology borrowed from the experimental analysis of behavior revealed that a low phenylalanine diet was unsuccessful in producing significant desirable behavioral changes in four subjects with which proper experimental controls were employed. However, when behavior therapy techniques were used with one of these same subjects, a rapid and dramatic reduction in stereotypy and self-abuse was observed.

\section{REFERENCES AND NOTES}

1. Ambrose, J.: A shortened method for the fluorometric determination of phenylalanine. Clin. Chem., 15: 15 (1969).

2. Anderson, V., Siegel, E., and Bruhl, H.: Behavioral and biochemical correlates of diet change in phenylketonuria. Pediat. Res., 10: 10 (1976).

3. Anderson, V., Siegel, F., Fisch, R., and Wirt, R.: Responses of phenylketonuric children on a continuous performance test. J. Abnorm. Psychol., 74: 358 (1969).

4. Ayllon, T., Layman, D., and Kandel, H. J.: A behavioral-educational alternative to drug control of hyperactive children. J. Appl. Behav. Anal., 8: 137 (1975).

5. Baer, D. M., Wolf, M. M., and Risley, T. R.: Some current dimensions of applied behavior analysis. J. Appl. Behav. Anal., 1: 91 (1968).
6. Barlow, D., and Hersen, M.: Single-case experimental designs. Arch. Gen. Psychiat., 29: 319 (1973).

7. Barrett, B. H.: Behavior analysis. In: J. Wortis: Mental Retardation and Developmental Disabilities, Vol. 8. (Brunner/Mazel, New York, 1977).

8. Berman, J. L., and Ford, R.: Intelligence quotients and intelligence loss in patients with phenylketonuria and some variant states. J. Pediat., 77: 764 (1970).

9. Bijou, S. W., Peterson, R. F., and Ault, M.: A method to integrate descriptive and experimental field studies at the level of data and empirical concepts. J. Appl. Behav. Anal., 1: 175 (1968).

10. Birnbrauer, J.: Mental retardation. In: H. Leitenberg: Handbook of Behavior Modification and Behavior Therapy (Prentice-Hall, Englewood Cliffs, NJ, 1976).

11. Bostow, D., and Bailey, J.: Modification of severe disruptive and aggressive behavior using time-out and reinforcement procedures. J. Appl. Behav. Anal., 2: 31 (1969).

12. Bruhl, H., Arneson, J., and Bruhl, M.: Effect of a low-phenylalanine diet on older phenylketonuric patients. Amer. J. Ment. Defic., 69: 225 (1965).

13. Corte, H., Wolf, M., and Locke, B.: A comparison of procedures for eliminating self-injurious behavior of retarded adults. J. Appl. Behav, Anal., 4: 201 (1971).

14. Ferster, C. B., Culbertson, S., and Perrott-Boren, M. C.: Behavior Principles (Prentice-Hall, Englewood Cliffs, NJ, 1975).

15. Fisch, R., Torres, F., Graver, H., Greenwood, C., and Anderson, J.: Twelve years of experience with phenylketonuria. Neurology, 19: 659 (1969).

16. Frankenburg, W. K., Goldstein, A. D., and Olson, C. D.: Behavioral consequences of increased phenylalanine intake by phenylketonuric children: A pilot study describing a methodology. Amer. J. Ment. Defic., 77: 524 (1973).

17. Fuller, R., and Shuman, J.: Treated phenylketonuria: Intelligence and blood phenylalanine levels. Amer. J. Ment. Defic., 75: 539 (1971).

18. Hackney, I., Hanley, W., Davidson, W., and Lindsao, L.: Phenylketonuria: Mental development, behavior, and termination of diet. J. Pediat., 72: 646 (1968).

19. Hambraeus, L., Holmgren, G., and Samuelson, G.: Dietary treatment of adult patients with phenylketonuria. Nutr. Metabol., 13: 298 (1971).

20. Hawkins, R. P., and Dotson, V. A.: Reliability scores that delude: An Alice in Wonderland trip through the misleading characteristics of interobserver agreement scores in interval recording. In: E. Ramp and G. Semb: Behavior Analysis: Areas of Research and Application. (Prentice-Hall, Englewood Cliffs, NJ, 1975).

21. Hersen, M., and Barlow, D. H.: Single Case Experimental Designs: Strategies for Studying Behavior Change. (Pergamon Press, New York, 1976).

22. Hudson, F.: Termination of dietary treatment of phenylketonuria. Arch. Dis. Childhood, 42: 198 (1967).

23. Kanfer, F. H.: Self-control in children: Research and theory. Presented at the Symposium on Learning and Behavior Modification in Educational Areas, Madrid, Spain, January 1975.

24. Kanfer, F. H.: Self-management methods. In: F. H. Kanfer and A. P. Goldstein: Helping People Change. (Pergamon Press, Elmsford, NY, 1975).

25. Kanfer, F. H., and Karoly, P.: Self-control: A behavioristic excursion into the lion's den. Behav. Ther., 3: 398 (1972).

26. Kang, E., Kennedy, J., Gates, L., Burwash, I., and McKinnon, A.: Clinical observations in phenylketonuria. Pediatrics, 35: 932 (1965).

27. Kazdin, A. E.: Behavior Modification in Applied Settings. (Dorsey Press, Homewood, IL, 1975).

28. Kazdin, A. E., and Marholin, D., II.: Program evaluation in clinical and community settings. In: D. Marholin II: Child Behavior Therapy (Gardner Press, New York, 1978).

29. Knox, W.: Phenylketonuria. In: J. Stanbury, J. Wyngaarden, and D. Fredrickson: The Metabolic Basis of Inherited Disease (McGraw-Hill, New York, 1976).

30. Levy H. L.: Newborn screening for metabolic disorders. N. Engl. J. Med., 288: 1299 (1973).

31. Liberman, R. P., Davis, J., Moon, W., and Moore, J.: Research design for analyzing drug-environment-behavior interaction. J. Nerv. Ment. Dis., 156: 423 (1973).

32. Lichstein, K., and Wahler, R.: The ecological assessment of an autistic child. J. Abnorm. Child Psychol., 4: 31 (1976).

33. Lonsdale, D., and Faust, M.: Normal mental development in treated phenylketonuria. Am. J. Dis. Child., 119: 440 (1970).

34. Marholin, D., II, and Bijou, S. W.: Behavioral assessment: Listen when the data speak. In: D. Marholin II: Child Behavior Therapy (Gardner Press, New York, 1978).

35. Marholin, D., II, Siegel, L. J., and Phillips, D.: Transfer and maintenance: A search for empirical procedures. In: M. Hersen, R. M. Eisler, and P. M. Miller: Progress in Behavior Modification, Vol. 3 (Academic Press, New York, 1976).

36. Marholin, D., II, Touchette P., and Stewart, R. M.: The effects of chlorpromazine withdrawal on the behavior of retarded adults (Manuscript in review, 1978).

37. Mash, E. J., and Makohuniuk, G.: The effect of prior information and behavioral predictability on observer accuracy. Child Dev., 55: 513 (1975).

38. McKean, C.: Effects of a totally synthetic, low-phenylalanine diet on phenylketonuric patients. Arch. Dis. Childhood, 46: 608 (1971).

39. O'Flynn, M.: Diet therapy in phenylketonuria: How long should it continue? Amer. J. Nurs., 67: 1658 (1967).

40. O'Leary, K. D., and Kent, R. N.: Sources of biases in observer recordings. 
In: B. Etzel, M. LeBlanc, and D. M. Baer: New Dimensions in Behavioral Research: Theory, Methods, and Applications. (Lawrence Erlbaum Associates, Hillsdale, NJ, 1977).

41. O'Leary, D. K., Kent, R. N., and Kanowitz, J.: Shaping data collection congruent with experimental hypothesis. J. Appl. Behav. Anal., 8: 43 (1975).

42. Olson, C.: Measurement of behavioral changes in phenylketonuric children attributed to dietary treatment (Unpublished doctoral dissertation, University of Arizona, 1968).

43. Sidman, M.: Tactics of Scientific Research (Basic Books, New York, 1960).

44. Siegel, L. J., Dragovich, S. L., and Marholin, D., II: The effects of biasing information on behavioral observations and rating scales. J. Abnorm. Child. Psychol., 4: 221 (1976).

45. Skinner, B. F.: Science and Human Behavior (Macmillan, New York, 1953).

46. Solomons, G., Keleske, K., Opitz, E.: Evaluation of effects of termination of the diet in phenylketonuria. J. Pediat., 69: 596 (1966)

47. Steinhauser, H. C.: Psychological evaluation of treatment in phenylketonuria: Intellectual, motor, and social development. Neuropaediatrie, 5: 146 (1974).

48. Stewart, R. M., Hemli, S., Daniels, G., Kolodny, E. H., and Maloof, F.: The pituitary-thyroid axis in adults with phenylketonuria. J. Clin. Endocrinal. Metab., 42: 1179 (1976).

$0031-3998 / 78 / 1203-0179 \$ 02.00 / 0$

Copyright $\odot 1978$ International Pediatric Research Foundation, Inc.
49. Stewart, R. M., and Kolodny, E. H.: Carbohydrate metabolism in adult phenylketonuria. (Unpublished manuscript, Eunice Kennedy Shriver Center, 1977).

50. Y-Hsia, D., Knox, W., Quinn, K., and Paine, R.: A one-year controlled study of the effect of low-phenylalanine diet on phenylketonuria. Pediatrics, 24: 148 (1958).

51. Zlutnick, S., Mayville, W. J., and Moffat, S.: Modification of seizure disorders: The interruption of behavioral chains. J. Appl. Behav. Anal., 8: 1 (1975).

52. The authors would like to recognize the significant contribution of the following individuals: Nancy Clark and Julia Sidel, dieticians at the Walter E. Fernald State School; Debra Lesco, who collected a significant portion of the data in experiment I; Debra Goodman, who served as trainer in experiment II; Mary Diogo, for her assistance in collecting data in experiment I; and Amy Feldman, who exhibited unbelievable patience in typing the manuscript.

53. This research was supported in part by USPHS Grants HD 05515 and HD 04147.

54. Requests for reprints should be addressed to: David Marholin II, Ph.D., Boston University, 765 Commonwealth Ave., Boston, MA 02215 (USA).

55. Received for publication March 7,1977.

56. Accepted for publication June 10, 1977. 